\title{
Agua-Territorio en América Latina: Contribuciones a partir del análisis de estudios sobre conflictos hídricos en Chile
}

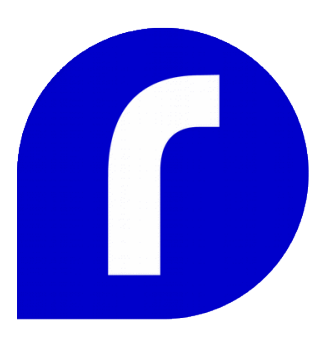

Recibido: 11 de agosto 2017

Revisado: 17 de octubre 2017

Aprobado: 16 de noviembre 2017

Alexander Panez Pinto

Chileno. Doctorando en

Geografía, Programa de

Post-graduación en

Geografía, Universidad

Federal Fluminense (Brasil).

Investigador del

“Laboratório de Estudos de

Movimentos Sociais e

Territorialidades" (LEMTO-

UFF). Con experiencia de

investigación en las

temáticas de cuestión

urbana y derecho a la

ciudad, y sobre re-

apropiaciones del agua y

conflictos territoriales.

Correo electrónico:

alexander.panez@gmail.com

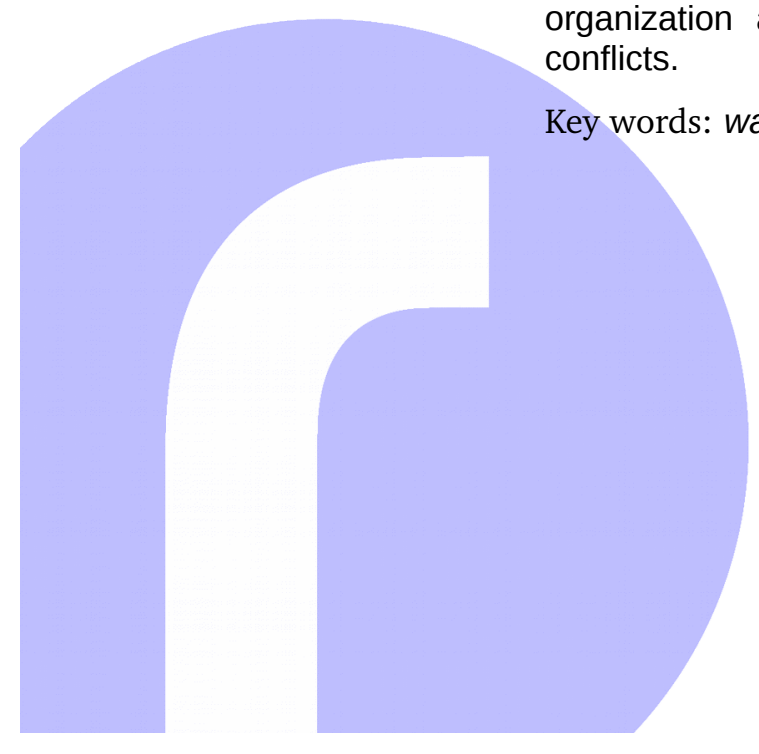

http://investiga.uned.ac.cr/rupturas/ (c) (1)(2) conflicts.
Resumen: En este artículo se reflexiona sobre las contribuciones que una lectura territorial entrega al análisis sobre conflictos por agua en América Latina. Tomando como caso la producción académica en Chile, revisamos investigaciones sobre conflictos hídricos en este país, identificando diferentes perspectivas de comprensión de la dimensión espacial de esta problemática. Discutiendo referencias de la ecología política y la geografía crítica, proponemos una construcción teórica sobre el agua pensada articuladamente del territorio como categoría teórico-política. Esta reflexión sobre aguaterritorio se compone de cuatro principios: a) comprender la vinculación cultura-naturaleza en los procesos de apropiación del agua, b) las relaciones de poder "en" y "a través" del territorio, c) las lógicas de organización espacial, y d) reconocer las otras territorialidades presentes en los conflictos.

Palabras clave: agua; territorio; América Latina; conflictos territoriales; Chile

\section{Water-Territory in Latin America: Contributions from the Analysis of Water Conflicts Studies in Chile}

Abstract: This article reflects about the contributions that a territorial interpretation gives to the analysis of water conflicts in Latin America. Taking as a case study the academic literature in Chile, it presents a review of the researches about water conflicts, identifying different perspectives of understanding the spatial dimension of this problem. Analyzing references from political ecology and critical geography, we propose a theoretical construction on water articulated with territory as a theoretical-political category. This analysis on water-territory is composed by four principles: a) to understand the nature-culture relation in the processes of water appropriation, b) the power relationships "in" and "trough" the territory, c) the logics of spatial organization and d) to recognize the other territorialities present in the

Key words: water; territory; Latin America; territorial conflicts; Chile 


\section{Introducción}

Las problemáticas asociadas con el agua en América Latina se han hecho más evidentes en los últimos años. La dificultad o el inminente riesgo en el acceso al agua para consumo humano de millones de personas se han visibilizado progresivamente en la agenda pública. La región se caracteriza por la paradoja de que a pesar de tener el mayor volumen de agua dulce per cápita del mundo con $22 \mathrm{mil} \mathrm{m}^{3}$ por habitante anual (el promedio mundial es de poco más de $6 \mathrm{mil} \mathrm{m}^{3}$ ), una parte considerable de la población del continente tiene acceso precario a este bien común natural (Peña, 2016).

Tal precarización en la disponibilidad de agua es determinada por las consecuencias de las formas dominantes de apropiación de aguas en articulación con las actividades de explotación minera, energía, agricultura de exportación y plantaciones forestales. Las consecuencias de esa articulación son la expresión de las paradojas del modelo de desarrollo adoptado en los países de la región, que presenta como uno de sus pilares generar crecimiento mediante procesos de despojo de territorios (Bartra, 2016).

La situación descrita lleva a que en América Latina proliferen las investigaciones que abordan la cuestión de los conflictos territoriales en que el agua adquiere centralidad, con base en diferentes perspectivas de análisis (Yacoub et al., 2015; Larsimont y Grosso, 2014; Castro, 2016). Sin embargo, concordamos con lo que afirman autores de la ecología política: a pesar de su evidente componente socionatural, la reflexión teórica sobre la vinculación entre las problemáticas asociadas al agua y las transformaciones territoriales en sus diferentes dimensiones, requiere ser profundizada con enfoques que complejicen la relación agua-sociedad (Larsimont y Grosso, 2014). Para avanzar en esta dirección, nos proponemos analizar la producción académica sobre conflictos por agua a partir de sus lecturas sobre el territorio y los procesos de territorialización. Dicha propuesta nos parece importante, ya que, como intentaremos argumentar a lo largo del texto, una comprensión densa del territorio nos puede permitir enfrentar de forma más compleja las disputas existentes por la apropiación del agua.

Para llevar a cabo esta reflexión, tomamos como caso de estudio la literatura académica sobre agua y conflictos en Chile. La elección de este país resulta relevante por las crecientes problemáticas relativas al agua (con más fuerza en la última década) y la visibilidad política que la cuestión hídrica ha adquirido en el país. Diferentes autores apuntan a que el intrincado escenario sobre el tema hídrico en el país se vincula con el radical proceso de neoliberalización que Chile experimentó luego del golpe de Estado de 1973 (Budds, 2013). En el caso del agua, fue elaborada una inédita legislación promulgada en 1981, que representa una experiencia única a nivel mundial de radicalización de la lógica mercantil (Bauer 2015). El código de aguas creado, privatiza este bien común no solo en lo que se refiere a su abastecimiento (como en el caso de los empresas sanitarias), sino que también posibilita y promueve la 
propiedad privada de las fuentes de agua en sus diferentes usos (agrícola, hidroeléctrico, minero, etc.).

El código de aguas se sustenta en cuatro pilares; (1) el código contiene una contradicción que termina por priorizar su uso como bien económico. Mientras que en su inicio se señala que el agua es un bien nacional de uso público, a lo largo de sus artículos fortalece la propiedad privada y el control privado de los derechos de agua, lo que es consagrado a nivel constitucional; (2) el Estado concede derechos de agua gratuitos y perpetuos a privados, los que pueden comprar, vender, hipotecar o arrendar estos derechos sin intervención del Estado; (3) separa la propiedad del agua del dominio de la tierra, lo que permite que existan privados que tienen acceso al agua sin necesidad de contar con tierra, mientras que existe un número creciente de campesinos que, a pesar de tener tierra, no tienen agua; (4) crea dos tipos de derechos de agua, que son los derechos consuntivos - de consumo, ya sea para actividades económicas, como para uso doméstico; y los derechos no consuntivos -que se usan pero no se consumen, como por ejemplo el caso de la generación de hidroelectricidad.

En este contexto, se vive en el país un aumento de los conflictos territoriales donde el agua adquiere centralidad, donde las comunidades locales se han organizado y exigido alternativas a las actividades extractivas de las empresas y el Estado. Solamente en el 2013, la iniciativa "Agua que has de Beber" contabilizó un total de 47 conflictos por ese líquido; se trata de una cifra considerable en un país como Chile. Los casos más críticos son aquellos ubicados en la zona norte-centro del país, donde la población hace casi una década está siendo afectada negativamente por una fuerte sequía agravada por la explotación de minerales y las grandes plantaciones de monocultivos. Son conflictos que han explicitado las consecuencias de la apropiación desigual del agua para el consumo humano, al igual que para la reproducción de las condiciones materiales de vida de las comunidades que dependen de este bien común para subsistir (sobre todo campesinos e indígenas). Nos llama la atención que en estas resistencias, aparecen sentidos diferentes sobre la apropiación del agua; pero también acerca del estar en la tierra-territorio (incluso otras ontologías como discutiremos posteriormente).

En sintonía con esta situación, el interés por estudiar los conflictos por el agua en Chile ha sido creciente, lo que se refleja en el aumento considerable de investigaciones (Prieto, 2016; Budds, 2012; Bauer, 2015; Larraín, 2006). No obstante, consideramos que la vinculación entre el agua y los procesos de territorialización es incipiente en la mayoría de esos trabajos, como intentaremos demostrar después.

Para efectos del análisis que sintetizamos en este artículo, vale la pena aclarar que, más que centrarnos en referencias empíricas de conflictos que involucran la cuestión hídrica en Chile, nuestro foco radica en un análisis teórico sobre cómo están siendo investigados este tipo de conflictos y, particularmente, cuál es la comprensión sobre la relación agua-territorio que subyace en la producción intelectual. Antes de comenzar con la exposición, alguien se podría aventurar a preguntar: ¿por qué es tan importante pensar los conflic- 
tos por agua en su relación con el territorio? Aquí ya se nos presenta un primer cuestionamiento. Podemos apresurarnos y responder que la inclusión del concepto de territorio en el análisis de los conflictos por agua se justifica en la ausencia de su reflexión en las investigaciones que se han llevado a cabo en América Latina. Una constatación preliminar, a partir del debate sobre los conflictos por agua en la región y en Chile como foco de reflexión, es que la categoría de territorio no ha sido colocada con la fuerza suficiente en el debate para problematizar esas disputas. No obstante, más que una ausencia, sostenemos que ha tenido una presencia limitada, ya que parte de estos estudios realizan una lectura espacial (muchas veces restringida desde nuestro punto de vista) sobre la problemática hídrica, independientemente de que utilicen o no el término territorio. Por este motivo, nuestro estudio comenzó con la revisión de producciones académicas realizadas sobre la problemática hídrica en Chile (plasmadas en artículos, informes, libros. etc.), realizadas desde diferentes disciplinas tales como: antropología, ciencia política y geografía, derecho, entre otras.

Nuestro análisis de estas elaboraciones se centró en rastrear las huellas de la reflexión sobre territorio en la discusión sobre conflictos por agua en el país. A partir de esta revisión, identificamos tres perspectivas en la relación agua-territorio presente dentro de ese debate. Sin embargo, el cuestionamiento incómodo puede persistir; digamos que estamos de acuerdo en que la reflexión sobre el territorio tiene una presencia limitada en los conflictos por agua. Pero aun así, ¿por qué es importante que esté presente? Para avanzar en esa interrogante, se plantea así la necesidad de argumentar sobre las contribuciones que el concepto de territorio nos puede entregar para entender de mejor forma los conflictos por agua en Chile. No obstante, antes de comenzar con nuestras argumentaciones sobre la importancia del territorio, se nos presenta la dificultad de que esta palabra es una categoría ampliamente discutida y polisémica en sus comprensiones (Haesbaert, 2014). Por lo tanto, ¿qué concepción de territorio? En este punto, consideramos que son provocadores los aportes del pensamiento crítico sobre el territorio producido desde la geografía como disciplina y la ecología política como campo de confluencia (Porto-Gonçalves, 2002; Escobar, 2014; Haesbaert, 2014; Souza, 2013; Santos, 1994). Por esta razón, en un segundo apartado hacemos una breve exposición de referentes teóricos que discuten sobre territorio desde una perspectiva crítica, con énfasis en aquellos que han profundizado sus análisis a partir de las experiencias de los movimientos sociales.

Hecho el recorrido descrito, estaremos en mejores condiciones para retomar uno de los cuestionamientos iniciales: ¿por qué es importante pensar los conflictos por agua en Chile y el resto de América Latina en su relación con el territorio?. De esta forma, reflexionamos sobre los aportes y las limitaciones de la perspectiva teórica y política sobre el territorio que proponemos para la discusión actual sobre conflictos por agua. 


\section{Huellas espaciales en los estudios sobre conflictos por agua}

En este apartado hablo de huellas espaciales para no remitir nuestra atención únicamente a aquellos trabajos en los que el concepto del territorio esté explícitamente colocado. Concordamos con Haesbaert en que el término territorio forma parte de una constelación de conceptos en torno a la categoría clave de espacio (Haesbaert, 2014). El territorio sería la expresión de un énfasis en ciertas propiedades y dimensiones del espacio, las que serán privilegiadas según las problemáticas que buscamos enfrentar. Por ende, nuestro objetivo será rastrear las lecturas espaciales presentes en las investigaciones sobre conflictos por agua en Chile.

A partir de la literatura revisada, identificamos diferentes formas de analizar la problemática del agua y su vinculación con la dinámica espacial, presentes en los estudios. De esta forma, se propone diferenciar las siguientes tendencias:

a) Existen investigaciones que se centran en aspectos físicos (geomorfología, precipitación, temperatura, etc.) de las transformaciones en la disponibilidad de agua; se relacionan con la problemática del cambio climático y las consecuencias de la demanda hídrica en las cuencas (Centro de Ciencia del Clima y la Resiliencia 2015, Núñez et al., 2011). Podemos reconocer una lectura espacial que enfatiza la dimensión material y "natural" del espacio donde suceden los conflictos por agua.

Cuando el Centro de Ciencia del Clima y la Resiliencia analiza la Ilamada megasequía que afectó sobre todo a la zona central del país durante el periodo 2010-2015, en lo referido a la intervención humana se remite a señalar que "el cambio climático antrópico es responsable de al menos un cuarto del déficit observado, una fracción que, se prevé, aumentará en el futuro." (Centro de Ciencia del Clima y la Resiliencia 2015, 22). No obstante, reducen la acción humana al cambio climático antrópico, que comprenden como el aumento en la emisión de gases efecto invernadero y disminución del ozono estratosférico, invisibilizando otros factores que inciden el proceso de sequía en el país, como la sobreexplotación de derechos de aguas en las cuencas ${ }^{1}$ y la proliferación de actividades de alto impacto en las fuentes hídricas (minería, forestal, agrícola, etc.). Además, al señalar "acción antrópica" se homogenizan responsabilidades como especie, sin distinguir las desiguales capacidades de impacto que los diferentes grupos y clases sociales poseen.

Consideramos que esta tendencia tiene una aproximación espacial restringida, al destacar los aspectos materiales, mientras le da poca importancia al carácter relacional de las acciones que los actores realizan y el espacio en el que estos interactúan. Tal visión se asemeja a la idea de espacio como un "contenedor" de relaciones (Montañez y Delgado, 1998) o substrato material (Souza, 2013). Se aprecia un énfasis en la dimensión física de la problemática del agua, restando importancia a aspectos sociopolíticos que afectan el "ciclo hidrológico". No es casual que en estos análisis sea más frecuente hablar de "problemática" más que de "conflicto" por agua. Justamente, el término conflicto hace alusión a una "expresión de las tensiones y contradiccio-
1. Según la Política Nacional de Recursos Hídricos, hasta el 2015 la Dirección General de Aguas ha sobreotorgado 110 acuíferos en todo el país (Ministerio del interior, 2015) 
nes del propio orden social que constituye en la propia medida que transforma" (Porto-Gonçalves, 2003, 3. Traducción nuestra), dimensión que permanece esbozada débilmente en dicho tipo de estudios.

b) En parte de los estudios sobre los conflictos por agua desde las ciencias sociales, se aprecia en sus preocupaciones una tendencia a-espacial que se relaciona con la larga trayectoria de encuentros y desencuentros de las ciencias sociales con el espacio. Históricamente, esa tendencia en las ciencias sociales afirma que la sociedad no se escribe fuera del espacio, y que no existe sociedad a-espacial, sino que el espacio es social, como recuerda Milton Santos (2006).

En el caso de los conflictos por agua en Chile, habría que distinguir entre estos análisis provenientes de las ciencias sociales. Por una parte, se pueden identificar investigaciones que relevan aspectos simbólicos de la relación que los actores establecen sobre el agua y la problemática en torno a esta, como es el caso del trabajo de Skewes y Silva, referente a las narraciones sobre el agua en la cosmovisión mapuche (2007). El "sistema de objetos" (para utilizar la terminología de Milton Santos) es relevada en la medida en que tales objetos adquieren un significado para los grupos sociales. Esto se asemeja a la comprensión del territorio desde una vertiente cultural (Haesbaert ,2011), que tradicionalmente ha sido abordada por la antropología.

Por otro lado, en el ámbito de las ciencias sociales es posible identificar otro grupo de investigaciones sobre conflictos por agua que analizan la asimetría de las relaciones de poder entre los actores involucrados y las políticas del Estado que inciden en la privatización y la concentración de la propiedad de este bien común, como el estudio de Larraín (2006). En este tipo de trabajo, se evidencia lo señalado por Elden: la teoría política carece de un sentido de territorio (2010), ya que el impacto de las relaciones de poder dominantes en las dinámicas de cuencas, valles y comunidades se invisibiliza entre los análisis de la economía y la política.

Ambos tipos de trabajos comparten el rasgo de estar próximos al riesgo de enfocarnos en las relaciones sociales, en tanto se subvaloran la materialidad en la reflexión sobre cómo dichas relaciones son espacializadas y, a su vez, cómo el espacio condiciona las posibilidades de despliegue de estas relaciones sociales. Frente a ello, nos parece fundamental el rescate de la dimensión material, "sobre todo en un momento como el que vivimos en que se da cada vez más importancia a la dimensión simbólica, casi siempre de modo unilateral, como si lo simbólico fuera opuesto a lo material" (Porto-Gonçalves, 2002, 230. Traducción nuestra).

c) Enfoques que cuestionan la relación sociedad-naturaleza, en las que se busca superar la separación entre lo físico y lo simbólico. En este último grupo, se ubica las contribuciones de la ecología política, sobre todo algunos autores influenciados por la corriente anglosajona, quienes se han dedicado a estudiar de forma más específica las problemáticas en torno al agua, como los trabajos de Torres et al. (2016), Budds (2012) o Romero et al. (2009). 
Esta tendencia cuestiona la idea de agua vigente en las ciencias y los instrumentos de planificación, al afirmar la premisa de que:

El agua internaliza las relaciones sociales y políticas, contrario a ser meramente el objeto de la política [...] Nosotros buscamos trascender las categorías dualísticas de 'agua' y 'sociedad', y emplear una aproximación dialéctico-relacional para demostrar cómo las instancias de agua son producidas y cómo el agua producida reconfigura relaciones sociales (Linton y Budds 2014, 171. Traducción nuestra).

Esta condición del agua como producto y productora de relaciones sociales, que retoma la clásica idea de Lefebvre del espacio como producto y producción (2013), permite llevarnos al reconocimiento de las relaciones de poder que están detrás de ese proceso de producción. Esto lleva a Swyngedouw a señalar que la movilización del agua para diferentes usos en lugares diversos es un proceso cargado de conflicto, en que "cada sistema tecno-social para organizar el flujo y transformación del agua (a través de represas, canales, tuberías y similares) muestra cómo el poder social es distribuido en una sociedad determinada" (2009, 57. Traducción nuestra).

Sus contribuciones han sido interesantes para el debate en Chile (y América Latina), donde han incorporado nuevos términos como el de ciclo hidrosocial, en oposición al concepto tradicional de ciclo hidrológico. Este término sugiere que, además de estudiar cómo el agua fluye dentro del ambiente físico (atmósfera, superficie y subsuelo), también debemos considerar cómo este bien común es manipulado "por los involucrados sociales y las instituciones, a través de factores tales como obras hidráulicas, legislación, instituciones, prácticas culturales y significados simbólicos" (Budds 2012, 170).

De las tendencias presentadas, nos parece que estas aproximaciones son las que han logrado una lectura espacial más compleja sobre los conflictos por agua, al destacar la importancia de las relaciones de poder no solo en el acceso a este bien común, sino también en nuestra propia forma de comprensión respecto del agua. Por este motivo, el diálogo crítico con esta perspectiva será retomado en los apartados siguientes.

\section{¿Qué concepción de territorio?}

Como decíamos anteriormente, el concepto de territorio posee una pluralidad de significados. Incluso, en la actualidad nos encontramos en un punto en que este término es usado ampliamente en algunos círculos (planificadores de políticas públicas, movimientos sociales, fuerzas armadas y científicos, 
entre otros), muchas veces de manera irreflexiva. Se evidencia, pues, la vitalidad del concepto al no quedar capturado por los círculos académicos, sino que es utilizado ( $y$ en definitiva, apropiado) por diferentes grupos de la sociedad.

Antes de diferenciar perspectivas sobre el territorio, podemos afirmar que la mayoría de las acepciones existentes sobre este concepto hacen alusión y colocan un énfasis en la cuestión del poder sobre un determinado espacio. Como señala Raffestin, el territorio se puede entender como "una producción, a partir del espacio. Pero, la producción, por causa de todas las relaciones que involucra, se inscribe en un campo de poder" $(1993,144)$.

Ahora, las preguntas sobre cómo se comprende el poder y, por consiguiente, cómo ese poder se naturaliza, legitima, disputa y rechaza, serán fundamentales para diferenciar las miradas sobre el territorio. Esta relación intrínseca con el poder le da una condición al concepto de territorio que hace necesario apartarlo de la mera reflexión teórica y exige reconocer explícitamente su carácter político. La comprensión sobre el territorio de las diversas perspectivas existentes determinará nuestras representaciones sobre la legitimidad (o no) de las formas vigentes de organización como seres sociales y también nuestras propuestas para mantener, mejorar, transformar o incluso destruir dichas formas de organización. Por esto, la disputa por el significado del territorio se vuelve tan relevante. Dicho lo anterior, se nos presenta un escenario que exige un posicionamiento teórico y político. Como ya lo dijimos, para esos efectos, trabajaremos con algunas referencias del pensamiento crítico en torno al territorio.

Antes de comenzar el recorrido sobre dicha perspectiva, es fundamental explicar lo que se ha sido denominado como paradigma tradicional sobre el territorio. No nos detendremos en explicar esta concepción porque ya otros lo han hecho con más detalle (Haesbaert, 2011; Elden, 2010; y sobre todo Raffestin, 1993). Solo queremos mencionar que desde esta visión moderna (ampliamente difundida), el territorio se entiende como aquella área dominada por un Estado-nación, sobre la cual este ejerce su soberanía delimitada claramente por fronteras. En palabras de Ratzel, "sin territorio no se podría comprender el incremento de la potencia y de la solidez del Estado" (1990, 74. Traducción nuestra). Entonces, el territorio se conformaría por una fuerte raigambre jurídico-administrativa, se vincula con una centralización del poder que organiza el espacio y que además se relaciona directamente con la construcción cultural de una nación (y una territorialidad, podríamos decir).

Resultado de esta identificación del territorio igual a Estado en la historia moderna del concepto, se le dio una relevancia exacerbada a un tipo de poder en específico, lo que dificultó la separación de la utilización de la palabra territorio de las "necesidades ideológicas específicas vinculadas a la legitimación de esa fuente de poder, mediante la construcción de un discurso de enaltecimiento y de total privilegio analítico-conceptual del 'territorio patrio', del 'territorio nacional'" (Souza 2013, 92. Traducción nuestra). 
Muestra de lo anterior es que esta concepción influyó en quienes legitimaban (y continúan legitimando) este tipo de poder y en quienes se oponían a dicha hegemonía. La conquista del Estado, pensar como alternativa el reemplazar un poder político por otro o incluso crear un otro Estado para ejercer dominio por parte de un determinado grupo social fueron estrategias frecuentes de grupos en situación de subalternización durante los últimos siglos. Sin embargo, en las últimas décadas (sobre todo desde el comienzo de los años noventa) es posible apreciar una nueva fuerza en la discusión sobre el territorio en América Latina. Resulta interesante notar que ese impulso surge a partir de las acciones de movimientos sociales que han colocado la relevancia del territorio en escena (Porto-Gonçalves, 2002). Ejemplos son: el movimiento zapatista en México, el movimiento indígena en Ecuador y Colombia, o más recientemente el movimiento maya en Guatemala. Muchos de ellos han asumido características diferentes a otros movimientos surgidos históricamente en nuestra región, tanto en el terreno de las prácticas colectivas como de las formas de lucha y planteamientos reivindicativos, ya que frente a la desposesión "muchas de estas organizaciones se constituían en la identificación política de su desposesión (los sin tierra, sin techo), de las condiciones sobre las que se erigía la opresión (los pueblos originarios) o de la lógica comunitaria de vida amenazada" (Seoane, Taddei y Algranati, 2008, 6).

Tal irrupción alertó sobre los aspectos del territorio que no estaban siendo tomados en cuenta por la discusión llevada hasta ese momento. Es así que como crítica a la visión tradicional de territorio y en sintonía con las cuestiones colocadas por los movimientos, se posicionan otras visiones que Haesbaert agrupa bajo la denominación de "paradigma territorial contra-hegemónico". Dicho paradigma ve al territorio como un espacio vivido (en la idea de Lefebvre), conformado "por las múltiples relaciones sociales y culturales que hacen del vínculo sociedad-"tierra" (o naturaleza, si preferimos) un lazo mucho más denso" (Haesbaert, 2014, 54. Traducción nuestra). Estas reflexiones reconocen el componente ideológico en la confusión entre territorio y substrato espacial material (cosificación del territorio) y en la identificación exclusiva o casi exclusiva "del término territorio a un tipo de recorte político-espacial específico: aquel definido por el Estado-nación" (Souza 2013, 92).

Para el objetivo del presente texto, profundizaremos en las características de esta perspectiva relevantes para nuestra discusión. En primer lugar, un aspecto compartido con otras miradas sobre el territorio (principalmente las provenientes de la geografía), pero que es profundizado, corresponde a la importancia de la materialidad en vinculación con lo simbólico. La diferencia con otras perspectivas se concreta en el reconocimiento de la imbricación entre estas dos dimensiones; no se separa ni se percibe de manera estática ni lo material ni lo simbólico y ciertamente sin intentos de establecer causalidades unidireccionales. Por esto, se define el territorio como híbrido (Santos 1994) "entre sociedad y naturaleza, entre política, economía y cultura, y entre materialidad e 'idealidad', en una compleja interacción tiempo-espacio" (Haesbaert 2014, 67). 
Particularmente, nos parece importante poner en relieve la imbricación cultura/naturaleza inscrita en el territorio, que históricamente ha sido entendida de forma dicotómica por visiones oposicionistas en la trayectoria de la modernidad. Dicho dualismo ha sido cada vez más cuestionado, en la medida en que se ha dejado en evidencia cómo las nociones hegemónicas sobre la cultura y la naturaleza han sido socialmente construidas, al tiempo que ha invisibilizado otras formas de comprensión posibles (Ulloa 2011).

Ahora, ¿cómo se construyen dichas articulaciones que conforman la hibridez del territorio? Escobar afirma que se trata de dimensiones que se articulan en un "proceso de apropiación socio-cultural de la naturaleza y de los ecosistemas que cada grupo social efectúa desde su 'cosmovisión' u 'ontología"' $(2014,91)$. Esta referencia a la vinculación entre territorio y ontología será profundizada posteriormente.

En segundo lugar, una característica relevante es el redimensionamiento de la discusión sobre la cuestión del poder. Tal perspectiva refuerza y amplía la relación territorio-poder. La refuerza porque se reconoce que "no es apenas el territorio que solo puede ser concebido, concretamente, con la ayuda de la idea de poder [...] También el poder solo se ejerce con referencia a un territorio y, muy frecuentemente, por medio de un territorio" (Souza, 2013, 87). Por otra parte, amplia la concepción de poder en la medida que se entiende el territorio "a partir de la imbricación de múltiples relaciones de poder, del poder más material de las relaciones económico-políticas al poder más simbólico de las relaciones de orden más estrictamente cultural" (Haesbaert, 2014, 67).

Lo expresado anteriormente nos da otras posibilidades para enfrentar el debate sobre el territorio. Considerar el poder como una relación social más que un objeto y lugar específico, nos facilita comprender los mecanismos menos aparentes que fortifican esta relación en los procesos de territorialización. No solo la coerción por medio de muros o cercas, sino también el conjunto de representaciones que promueven naturalizar una determinada relación de dominación-apropiación (Lefebvre, 2013). Esta complejización del poder será un tema transversal en las reflexiones que siguen en este texto.

En tercer lugar, desde la perspectiva planteada, hay un reconocimiento de las lógicas de ordenamiento del espacio presentes, las cuales condicionan los flujos de objetos y el despliegue de las acciones de los diferentes actores en la conformación de los territorios. Haesbaert (2014) reconoce la existencia de una lógica de dominancia zonal (que privilegia delimitar, fijar acciones y controlar áreas) y otra reticular (que tiende a la expansión, circulación y a más fluidez) en la organización del espacio. Esta distinción nos permite comprender las lógicas de organización del espacio prevalecientes en las formas de dominación contemporáneas. De este modo, es posible apreciar cómo la configuración actual del territorio está pasando de un territorio más "'zonal'" (lógica típica del Estado-nación) para un 'territorio-red' o de control de redes (lógica típica de las grandes empresas). En ese punto, el movimiento o la movilidad pasa a ser un elemento fundamental en la construcción del territorio" (Haesbaert 2014, 68. Traducción nuestra). 
Se diferencia, por ejemplo, con las estrategias adoptadas dentro del propio capitalismo hace 200 años atrás, cuando la lógica zonal de ocupación colonial era más evidente en su forma de dominación. No queremos decir que la lógica zonal no continúe en pie; de hecho, en el caso de los conflictos por agua, su relevancia es explícita. Se trata de identificar, entonces, la historicidad de la relación entre ambas lógicas y cómo ocurre la simultaneidad entre ellas.

Además del reconocimiento de las lógicas de organización espacial, poner atención sobre los flujos o movimientos en la conformación de los territorios nos da la posibilidad de entender cómo la desigual relación de poder se traduce en una capacidad de incidencia diferenciada de las acciones que los diferentes actores despliegan sobre el espacio. En el caso de las clases dominantes, su mayor dominio de las redes espaciales que se tejen en el proceso de acumulación de capital, amplificado por su control sobre los medios de la tecnología actual, les da la capacidad de que sus acciones traspasen con una velocidad creciente el ámbito local en el que ellos mismos se desenvuelven cotidianamente.

Como señala Milton Santos, "varios puntos distantes son, al mismo tiempo, alcanzados, a partir de un mismo centro decisorio que envía sus mensajes y ordenes con eficacia" (2006, 149. Traducción nuestra). En aquellos territorios que van adquiriendo interés funcional para los actores hegemónicos y que históricamente han conformado los espacios vividos de otros grupos sociales, esta mundialización de sus acciones consolida un proceso de superposición "de acciones con escalas diversas, portadoras de contextos con diverso alcance geográfico y fuerza activa (o reactiva) diversa" (2006, 149. Traducción nuestra).

En el caso de los grupos subalternos, se va produciendo una alienación regional en la cual "las acciones son cada vez más extrañas a los fines propios del hombre y del lugar" (Santos, 2006, 51. Traducción nuestra). A partir de dicha constatación, el autor distingue entre la escala de realización de las acciones y la escala de su comando, razón por la que gran parte de las acciones desplegadas en un lugar son motivadas por necesidades ajenas (2006). El reconocimiento de esta capacidad desigual de movilización de nuestras acciones coloca en otra perspectiva el debate sobre la escala en el momento de estudiar los conflictos, pasando a comprenderla también como un concepto político. Como señala Smith, la escala no es únicamente una dimensión material abordada como paisaje; también "es la escala de resolución o abstracción que nosotros empleamos para entender las relaciones sociales, cualquiera que sea su impresión geográfica [...] De este modo, la escala de la lucha y la lucha sobre la escala son dos lados de la misma moneda" (Smith 2002, 142)

En cuarto lugar, la perspectiva tiene que ver con el énfasis en visibilizar las relaciones que los grupos subalternos establecen con los territorios. En este sentido, la pregunta que formula Arturo Escobar puede orientar el trasfondo de la atención sobre las otras territorialidades; 
¿Cómo se explica la tenacidad con la que muchas poblaciones y organizaciones locales, no solo luchan por defender sus territorios; sino que lo hacen a nombre de otra concepción del desarrollo, una relación armónica con la naturaleza y una forma diferente de vida social? (2014, 73).

Dicha tenacidad se puede intentar explicar por la profunda relación del territorio con la propia existencia de algunos grupos sociales. Tal vinculación, que en momentos de conflicto se puede volver dramática, se grafica con toda su potencia en la palabra de la siguiente líderesa comunitaria colombiana:

Nuestra apuesta es defender el territorio; nos quitaron la zona plana y nos fuimos para las laderas; ¿ahora para dónde nos corremos? La gente dice que es preferible morir de un tiro que irse a los corredores de miseria de las ciudades [...] Pero solamente volveremos a ser esclavos cuando el último hijo haya vendido el último metro de tierra (Lideresa comunitaria de La Toma, Cali; citada por Escobar, 2014).

Cuando ella señala que "volveremos a ser esclavos cuando el último hijo haya vendido el último metro de tierra", entendemos como trasfondo de que el territorio actual en el que viven les permite una cierta libertad, un despliegue de sus formas de vida. Esto implica una resignificación del concepto de territorio, buscando resolver su reivindicación histórica por tierra y al mismo tiempo, la demanda por el reconocimiento étnico-cultural como pueblos. De esta forma, el territorio como concepto pasa "a ser disputado por diversos grupos de la sociedad que poseen diferentes sentidos para estar en la tierra, indicando que no hay territorio sin territorialidad y, así, desnaturalizan ese debate llamando la atención para el proceso de apropiación del espacio geográfico - proceso de territorialización" (Porto-Gonçalves. 2017, 17. Traducción nuestra).

Este foco en el territorio desde los movimientos, que Porto-Gonçalves llama la atención como politización de la propia existencia (r-existencia), consideramos que es profundizada por Escobar cuando menciona que las territorialidades-territorializaciones de los movimientos se vinculan con una dimensión ontológica. El autor retoma la idea básica de ontología comprendida como nuestra forma de entender lo que significa que algo o alguien exista (Escobar 2014). Desde ahí, Escobar incorpora los trabajos de Mario Blaser sobre ontología política. Para nuestra argumentación, nos parece muy relevante la di- 
mensión práctica que estos autores le otorgan a la construcción de las ontologías, sacándolas de la discusión filosófica abstracta a la que suele remitirse como concepto. Así, las ontologías no solo se entienden "como imaginarios, ideas, o representaciones sino que se despliegan en prácticas concretas, prácticas que 'crean verdaderos mundos"' (Escobar 2014, 96)

En esta clave interpretativa, la pugna en curso entre los paradigmas territoriales señalados se trataría de un conflicto entre diferentes ontologías. Existe aquello que Escobar denomina "ontología dualista" sustentada en la modernidad dominante, que niega constantemente la existencia de otros mundos al apelar permanente a la idea de un uni-mundo en el cual existen diferentes culturas, pero una razón universal (Escobar, 2014). Otro componente de dicha ontología importante para nuestro trabajo, es la división cultura-naturaleza que se perpetúa. Esta separación sustenta otorgar una labor delimitada a disciplinas como la antropología como estudios de las diferentes culturas y a la política como devenir de lo humano, mientras que la verdadera ciencia debe investigar las cuestiones relativas al funcionamiento de la naturaleza (la cual no puede ser origen de hechos políticos). Para Escobar, los planteamientos de esa ontología dualista se amparan en cuatro "creencias fuertes" de la modernidad: creencia en el individuo, creencia en la economía, creencia en la verdad (ciencia), creencia en el mundo objetivo (real).

En contraposición a tal visión, las otras territorialidades mencionadas por nosotros, son denominadas como ontologías relacionales. En dichas ontologías, los territorios son comprendidos como "más que una base material para la reproducción de la comunidad humana [...] Cuando se está hablando de la montaña como ancestro o como entidad sintiente, se está referenciando una relación social, no una relación de sujeto a objeto" (Escobar 2014, 103). Si ampliamos este carácter relacional de la existencia fuera de los márgenes de una comunidad o pueblo específico, nos daría la posibilidad de entender la vinculación entre todos los entes y rincones del planeta. Se vislumbra la oportunidad de traspasar nuestra comprensión del uni-mundo y reconocer la presencia de un pluriverso.

Para finalizar el presente apartado, consideramos que Escobar nos provoca con algunas lecturas que nos pueden servir para repensar la relación entre territorio-poder. Es el caso de sus palabras sobre entender la cuestión de la autonomía como una instancia ontológica, como "capacidad de toda comunidad de definirse a sí misma, de fijar sus normas de existencia y convivencia, es decir, sus modos de r-existencia, y, en última instancia, de diseñarse a sí misma, en toda su diversidad y heterogeneidad y en su entramado con otros mundos" $(2014,123)$. 


\section{¿Por qué pensar la relación entre agua y territorio en los conflictos en curso?}

La inquietud por pensar la relación agua y territorio se basa en la preocupación del aislamiento analítico del agua como bien común en relación con el resto de los componentes presentes en el planeta para la reproducción de la vida. El movimiento que proponemos tiene que ver con darle una centralidad al agua dentro de las problemáticas del mundo contemporáneo debido a su creciente mercantilización por la lógica capitalista; y, además, por su presencia en "todo lo vivo", lo que le dan una condición diferente a otros bienes comunes naturales. Pero, al mismo tiempo, no queremos caer en su fetichización ni en su separación analítica, pues resulta riesgoso el foco exclusivo en el agua, desconectado de las múltiples relaciones que condicionan sus formas vigentes de dominación-apropiación. Hablamos de fetichización, tomando como inspiración la reflexión de Marx sobre el carácter fetichista de la mercancía (Marx 1996). En el caso del agua, la forma más radical de fetichización ocurre cuando este bien común asume la forma social de mercancía: aparece ante nosotros con características objetivas convertida en un producto intercambiable en el mercado, ajeno a las relaciones sociales entre quienes deben pagar para consumirla. Esta agua-mercancía, que parece independiente de las contradicciones de las relaciones entre los seres humanos, oscurece la vinculación de dicho bien común natural con el territorio desde el cual fue apropiada y la relación cultura-naturaleza que permite tal mercantilización. Adicionalmente, la fetichización del agua en tanto mercancía es un extrañamiento directo de nuestra existencia, que niega cómo el agua esconstituye vida. Sin embargo, hay formas más sutiles de fetichización, que invisibilizan las relaciones socionaturales detrás de este bien común natural. Ejemplo reciente es la tendencia de poner atención exclusiva en la comprensión del agua como objeto de investigación; es el caso de la proliferación de estudios, seminarios y encuentros que convocan a discutir sobre agua o el incremento exponencial en el mundo de especialistas sólo en la problemática del agua.

En este sentido, el aporte de Vargas y Piñeyro (2005) en su libro Hidroscopio es clave, al incorporar la premisa de que "el agua nunca es agua", sino que "es el uso que hacemos de ella lo que define, en última instancia, su significado. El para qué la quiero es lo que define lo que es el agua para mí" (2005, 11). Debido a esa condición escurridiza del agua, los autores nos invitan a pensarla de forma multidimensional mediante una "semiótica del agua" que nos permita "desmontar, a partir de la identificación de los diferentes campos de significados, las percepciones del agua que están incidiendo en la construcción de nuestra cultura hídrica" (Vargas y Piñeyro 2005, 80).

La separación analítica que comentamos, incluso puede afectar a los movimientos sociales. Por ejemplo, en el caso de Chile, donde se vive una realidad peculiar a causa de una legislación de aguas (Código de aguas de 1981), que en su propia formulación promueve la separación analítica del agua, no solo al abrir su apropiación como bien económico, sino que además separa la propiedad de la tierra del agua, permitiendo su libre transacción en 
el mercado sin las "ataduras" de una porción de tierra que determine su uso. Como ya señalamos, esto genera situaciones de campesinos e indígenas que cuentan con tierra para cultivar pero que no disponen del agua necesaria para sembrar en dicha tierra, sea porque no han regularizado sus derechos o porque tuvieron la necesidad de vender sus derechos a otros actores que tienen proyectos en el territorio. Este contexto, en el que la conflictividad en torno al agua en el país se ha vuelto tan explícita, ha incentivado a que movimientos sociales centren sus reivindicaciones en el derecho humano al agua, lo que conlleva el riesgo de llevar a reproducir la separación agua-tierra y contribuir a la invisibilización de temas centrales para la discusión de la defensa de los territorios, como es el caso de la concentración de la tierra en el país (según el censo agropecuario del 2007, 69,7\% de las tierras del país están agrupadas en explotaciones de más 2000 hectáreas; Echeñique, 2012).

Este movimiento de centralidad sin aislamiento analítico, considero que es un esfuerzo necesario en la dirección de generar una ruptura epistémica en las formas vigentes de comprensión del agua y los conflictos en torno a esta. Sobre todo, rebelarse contra la racionalidad hegemónica en la ciencia de la sociedad moderno-colonial que busca generar un saber altamente especializado y escindido del análisis de las relaciones sociales que configuran su apropiación. En este sentido, nos unimos a los esfuerzos de los autores de la ecología política en su empeño de cuestionar tanto disciplinas consolidadas, como es el caso de la hidrología, como paradigmas dominantes de gestión del agua como la llamada "gestión integrada de recursos hídricos" (Linton y Budds, 2010).

Como contribución para esta ruptura epistémica en las comprensiones actuales sobre el agua y sus flujos, proponemos el binomio agua-territorio para entender la centralidad de dicho bien común. El uso de este par no quiere decir que ambos sean equivalentes, sino que se refiere a la inseparabilidad que existe entre agua y territorio. Una conclusión que puede resultar obvia, pero que consideramos que no ha sido abordada densamente en la mayoría de los estudios actuales que tienden a parcelar su visión sobre el agua como elemento en conflicto.

Vale mencionar que en el campo de la Ecología Política, hay esfuerzos en el último tiempo por pensar la relación agua y territorio dentro de las investigaciones, lo que muestra la relevancia de pensar ambos aspectos de manera conjunta. Una de las producciones que contribuye de forma significativa al debate, es el reciente trabajo de Boelens, Hoogesteger, Swyngedouw, Vos y Wester (2016), "Hydrosocial territories: a political ecology perspective". En su artículo, los autores definen territorio hidrosocial como:

El disputado imaginario y materialización socio-ambiental de una red multi-escalar espacialmente vinculada en donde humanos, flujos de agua, relaciones ecológicas, infraestructura hidráulica, medios financieros, medidas lega- 
les-administrativas e instituciones y prácticas culturales son definidas, alineadas y movilizadas interactivamente a través de sistemas de creencias epistemológicas, jerarquías políticas y discursos naturalizadores (Boelens et al., 2016, 4. Traducción nuestra)

Nos parece que hay varios puntos de confluencia en la propuesta de los autores y la perspectiva que presentamos en este artículo, principalmente en lo referido a la vinculación entre agua y poder en los procesos de territorialización y la comprensión de la multi-escalaridad de esa dinámica. No obstante, nuestra divergencia está en la comprensión del territorio en su relación con las otras territorialidades, tal como será profundizado en el último punto de este apartado.

Resumiendo nuestro planteamiento, pensar el agua vinculada con el territorio implicaría comprender: a) la vinculación cultura-naturaleza presente en los procesos de apropiación del agua; b) las relaciones de poder desplegadas "en" y "a través" del territorio; c) las lógicas de organización espacial con la producción de escalas; y, finalmente, d) las diferentes territorialidades de los actores protagonistas de los conflictos en curso.

a) Vinculo Cultura-Naturaleza: comprender la relación cultura-naturaleza inscritas en la propia conformación de los territorios permite evitar las miradas del espacio como un substrato material, donde los fenómenos vinculados a la problemática del agua son tratados como aislados de las acciones antrópicas (diferenciadas y desiguales entre ellos). La forma actual de re-producción de la vida requiere una participación constante en la dinámica socio-metabólica de la naturaleza. No se trata de intervenciones humanas episódicas que son realizadas sobre un funcionamiento "normal" de la naturaleza, así como es presentado en la idea clásica de ciclo hidrológico. Esto implica desnaturalizar nuestras relaciones con la naturaleza, al comprender la mediación de la cultura y las formas de producción del espacio. Al mismo tiempo, también conlleva no subvalorar la dimensión biofísica que la cuestión hídrica posee. Las condiciones en las que el agua se presenta en los territorios (ríos, napas subterráneas y glaciares, etc.) configuran un paisaje que limita o posibilita sus formas de apropiación. Por lo tanto, comprender cómo el agua se va transformando y cómo interactúa con el resto de los procesos socionaturales continúa siendo una tarea fundamental.

b) Relaciones de poder: en su relación con el territorio, reflexionar sobre el poder es una forma de politizar la discusión sobre los conflictos en los que el agua aparece con centralidad, al relevar las disputas que hay en torno a este bien común.

Históricamente, el agua ha sido elemento de disputa e incluso mecanismo para ejercer dominación (como afirma el clásico trabajo de Karl Wittfogel), por lo que se inscribe en los procesos de tensión que conforman los múltiples 
territorios, y que condicionan las formas de apropiación posible de este bien común. Los planteamientos presentados tienen sintonía con los contribuciones de los autores que trabajan los conflictos por agua desde la ecología política (anglosajona, principalmente). Pero dicha politización no solamente sucede en razón de la disputa por un "recurso" que se vuelve "escaso" y codiciado, como afirmaremos posteriormente.

Otra cuestión importante de pensar el poder desde el territorio es que puede llevar a la necesaria reflexión sobre el poder al interior de los propios movimientos: ¿cómo estamos comprendiendo el poder? Y a partir de esto: ¿cómo estamos entendiendo nuestra relación con ese(os) poder(es)? Por ejemplo, y volviendo al caso chileno, resulta fundamental pensar sobre las alternativas para salir de la profunda privatización del agua como consecuencia del neoliberalismo consolidado en este país.

Dentro de los movimientos que luchan por el agua han surgido varias voces que exigen la renacionalización del agua. En las propuestas, se habla de la estatización de la gestión de este bien común fundamental, sin relevar una dimensión comunitaria en su funcionamiento. Sin embargo, ¿es realmente la alternativa que queremos? ¿Deseamos que el Estado vuelva a gestionar las aguas del país y que así consolide su control centralista y uninacional sobre el territorio?

Asimismo, pensar la relación territorio-poder es clave ya que puede significar un factor estratégico para la acción de los propios movimientos sociales. En este sentido, concordamos con lo afirmado por Porto-Gonçalves sobre la importancia de saber pensar y ocupar el espacio, ya que "al hacer el uso político del espacio a través de las estrategias espaciales, los protagonistas están rompiendo con un orden hegemónico moldeado en el derecho de la propiedad, del individuo, del derecho de ir y venir" $(2003,29)$.

Mingas, ocupación de calles o plazas, cortes de rutas, siembra de semillas criollas, denuncia (o cercamiento) de pozos ilegales y ceremonias en manantiales son algunas muestras de la ocupación-organización que los movimientos hacen del territorio. Muchas pueden ser repensadas y muchas otras pueden ser creadas. Lo importante es que los movimientos logren trascender el despliegue de esas acciones de forma aislada y consigan cada vez más pensarlas de forma articulada en el espacio.

c) Formas de organización espacial y producción de escalas: la visión trabajada de territorio nos permite reconocer la historicidad de las formas de organización del espacio para la dominación-apropiación del agua por parte de los actores hegemónicos. Muestra de ello es que en la fase actual del capitalismo se ejerza un dominio sobre los territorios articulando las lógicas zonal y reticular según sus objetivos de acumulación. Simultáneamente, se puede apreciar la acción de empresas transnacionales extractivas en América Latina que, en alianza con el Estado y fuerzas paramilitares, ocupan territorios de su interés y se apropian de los bienes comunes, entre ellos el agua (lógica de tipo zonal); y la articulación de los mercados financieros por medio 
de la red de ciudades-globales por la que circulan las inversiones de empresas sanitarias, de hidroelectricidad o de minería (de tipo reticular).

Además, pensar el territorio desde estas referencias nos permite profundizar la visión sobre las escalas de las relaciones que están actuando en los conflictos por agua. Estudiar la escala política de estos conflictos tiene que ver con reconocer el grado de intensidad política lograda por dichos conflictos. A partir de la comprensión de la capacidad diferenciada de las acciones de los actores para incidir en diferentes escalas, se abre un prolífico camino para desarrollar una metodología de estudio de los conflictos que permita analizar la capacidad de articulación y organización de sus protagonistas.

Nos hallamos, por lo tanto, ante una riqueza importante para el estudio de los conflictos por el agua, ya que la comprensión de estas escalas y los movimientos que realizan los actores en disputa permiten superar las visiones restringidas a la dimensión local del conflicto, que es justamente la escala a la cual el capitalismo nos quiere remitir. Y también, ir más de aquellas miradas que vinculan lo local con cuestiones de nivel nacional e internacional sin visibilizar las mediaciones por las cuales se da esa relación.

Pensar desde el territorio pone atención sobre las formas de organización socio-espacial, los flujos de circulación de mercancías, de la información, de las formas de articulación de las formas de dominación y de resistencia más allá de lo local. Esto nos propone como objetivo político de "superar la dominación social ejercida a través de la construcción explotadora y opresiva de la escala" (Smith 2002, 144); nos provoca a construir nuestra propias formas de organización espacial de la vida social, construir nuestra escala. Lo expresado es particularmente relevante para el caso del agua, la cual por su propia condición tiene la capacidad de escabullirse, diluirse y transformarse, por lo que pasan inadvertidas, a simple vista, sus múltiples relaciones.

d) Diferentes territorialidades en conflicto: finalmente, la mirada propuesta por el territorio reconoce que el conflicto puede existir entre diferentes ontologías. Hay una contribución central de la ecología política en América Latina, en que la preocupación por el "mirar desde abajo" marca una diferencia con los teóricos de la ecología política inglesa, de quienes se aprecia una poca atención a la comprensión de las "territorialidades otras" (que son relegadas a "prácticas culturales") involucradas en los conflictos por agua. Aquí radica nuestra principal diferencia con la conceptualización de "territorios hidrosociales" de Boelens et al. (2016), mencionada anteriormente. Los autores reconocen la existencia de un "pluralismo territorial" donde se confrontan y se suponen varios "territorios en un territorio", según las formas de vida y los significados sobre agua-naturaleza que los diferentes actores poseen. No obstante, nos parece que aún es limitado su énfasis sobre la profundidad ontológica que subyace en las territorialidades de algunos grupos sociales del sur global. El grito de "No queremos tierra, queremos territorio" implica más que un reconocimiento de la diversidad de territorialidades a partir de diferentes visiones de mundo (Boelens et al., 2016). Apunta, igualmente, a la reivindicación del territorio como horizonte político; una apuesta de presente y futuro 
para la construcción de otras formas de organización para la vida, ajenas a la comprensión moderno-colonial del territorio en la estructura del Estado.

Lo anterior interpela directamente a visiones ampliamente difundidas de la ecología política, que afirman que los conflictos "ecologico-distributivos" se configurarían entre diferentes "lenguajes de valoración" (Martínez-Alier 2014) o que se trata de una cuestión de asimetría del poder y diferencias culturales (en este caso, diferentes culturas del agua). En muchos casos de conflictos territoriales donde el agua adquiere importancia, apreciamos que se trata de cuestiones que van más allá de esta caracterización. Parte de esos conflictos, sobre todo aquellos en los cuales participan grupos campesinos, indígenas y afrodescendientes, no se tratan solo de agua, cultura o recursos. También están en conflicto las formas diferentes de entender la propia existencia de pensar el(los) mundo(s).

Esta atención hacia el mirar de los movimientos es relevante porque muchas veces estos son los grandes ausentes del conocimiento generado desde la academia sobre los conflictos por agua. En el caso de la producción sobre esta temática en Chile, los grupos subalternos suelen ser remitidos a su condición de "afectados", sin más capacidad creativa que la mera reacción y la oposición a los daños causados por un determinado proyecto.

\section{Conclusiones}

A partir de la revisión de producciones académicas realizadas en Chile sobre conflictos hídricos, nos cuestionamos en este escrito sobre las posibilidades que el concepto de territorio, concebido desde una perspectiva crítica, nos permite para pensar de forma más compleja las relaciones que están implicadas cuando hablamos de conflictos por agua. En este apartado, presentamos algunas limitaciones posibles por tener en cuenta para futuros trabajos y contribuciones que se abren para otras investigaciones a partir de las reflexiones presentadas.

Dentro de la academia, una limitación posible se refiere al riesgo de pasar de la completa negación de la territorialidad del otro y su ontología a la idealización de los movimientos y el cuestionamiento ontológico al unimundo moderno-colonial. En este sentido, ¿en qué medida esta atención concentrada en la territorialidad-territorialización de "los de abajo" puede acabar en idealizaciones de los procesos estudiados?

Uno de los principales aprendizajes de trabajos como los de Porto-Gonçalves con los seringueiros de Acre, al igual que los de Escobar con los afrocolombianos del Pacífico colombiano, es reconocer el valor de nuevas categorías para comprender la realidad a partir de la propia experiencia de los movimientos sociales y no al revés. Está presente el desafío de no forzar la lectura sobre la experiencia de los movimientos sociales a partir de nuevas categorías que nosotros consideramos interesantes o "avanzadas". 
En el caso de las luchas por agua en Chile, por un lado, apreciamos de que parte de los movimientos que protagonizan las disputas por agua en la actualidad no tienen un horizonte ontológico relacional en sus reivindicaciones, sino que se asimilan más a lo que ha sido caracterizado como prácticas NIMBY (Not in my backyard). Por otro lado, tenemos el ejemplo de los pueblos indígenas como el mapuche (Torres et al 2016) o el atacameño (Prieto, 2016), que cada vez más se han involucrado en conflictos donde el agua adquiere centralidad, con una relación ancestral que concuerda con lo que Escobar llama de ontologías relacionales. Esta diversidad nos exige ser cuidadosos al momento de interpretar la acción de los movimientos y comprender cuáles son los componentes que están configurando los conflictos estudiados (Azuela y Mussetta, 2009). Profundizar en el análisis empírico sobre las formas de resistencias en curso en Chile y su vinculación con la territorialidadterritorialización de los grupos en conflicto son acciones por tener en cuenta para futuras investigaciones.

A pesar de tal posible limitación, nos parece que esta construcción teórica contribuye a un incipiente camino de investigación. Particularmente, pensando en los aportes que se abren con este trabajo, a pesar de que el artículo toma como caso estudio la producción intelectual sobre Chile, nos parece que las reflexiones son pertinentes para otros países de América Latina. Especialmente, porque se aprecia que en el debate sobre conflictos hídricos en la región, el análisis sobre la relación entre agua y transformaciones socioespaciales es aún incipiente, a pesar de significativos aportes, como lo reflejan los trabajos de Boelens et al., 2016, Yacoub et al. 2015, Larsimont y Grosso, 2014. Nos parece que se presenta un desafío sobre qué profundizar. Nos encontramos en un vigoroso momento de investigación sobre conflictos territoriales y agua en América Latina, con un fuerte énfasis en los estudios de casos de conflictos en los países. No obstante, con la cantidad de producción académica existente, estamos en un punto en el cual consideramos que se puede avanzar más en la reflexión sobre cómo se están investigando tales conflictos en la región y cuáles son las perspectivas en que se sustentan estos estudios. Consideramos que interrogar este tipo de estudios, a partir de la lectura territorial que establecen, es un camino fecundo para problematizar la producción académica en América Latina.

Por otra parte, nos parece que los aportes de esta construcción analítica no se remiten solamente a la disputa epistémica-política en los espacios académicos. La mirada de conjunto propuesta en dicha conceptualización de territorio rescata un cúmulo de experiencias y reflexiones que pueden contribuir a la problematización que los movimientos sociales hacen respecto de los conflictos en torno a la apropiación del agua. Relacionar poder, multi-escalaridad y cultura-naturaleza de manera compleja puede apoyar a desnaturalizar las trampas analíticas de la racionalidad moderno-colonial que muchas veces acabamos reproduciendo en los espacios de resistencia (tales como los binarismos como agua / tierra y sociedad / naturaleza).

Por último, afirmamos que el llamado de la perspectiva propuesta a mirar los movimientos desde sus múltiples territorios y su capacidad de generar cono- 
cimientos y de reinventar categorías analíticas (en este caso, el concepto de territorio), se basa en la constatación de la potencialidad que estas agrupaciones tienen para enfrentar el proyecto vigente del capitalismo, empeñado en hacernos creer que solo existe un mundo: su mundo.

\section{Bibliografía}

Azuela, Antonio y Paula Mussetta. 2009. «Algo más que el ambiente. Conflictos sociales en tres áreas naturales protegidas de México». Revista de Ciencias Sociales 16: 1-25.

Bartra, Armando. 2016. «Con los pies sobre la tierra». En: Se hace terruño al andar. Las luchas en defensa del territorio de Armando Bartra, Carlos Walter Porto-Gonçalves y Milson

Bauer, Carl. 2015. Canto de Sirenas: El derecho de aguas chileno como modelo para reformas internacionales. Santiago de Chile: Ediciones El Desconcierto, 2015.

Betancourt. Ciudad de México: UAM-Xochimilco.

Boelens, Rutgerd; Jaime Hoogesteger, Erik Swyngedouw, Jeroen Vos y Philippus Wester. 2016. «Hydrosocial territories: a political ecology perspective». Water International 41 (1): 1-14.

Budds, Jessica. 2012. «La demanda, evaluación y asignación del agua en el contexto de escasez: un análisis del ciclo hidrosocial del valle del río La Ligua, Chile». Revista de Geografía Norte Grande 52: 167-184.

Budds, Jessica. 2013. «Water, power, and the production of neoliberalism in Chile, 1973-2005». Revista Environment and Planning D: Society and Space 31: 301-318.

Castro, José Esteban. 2016. Água e democracia na América Latina. Editora UEPB, Paraíba.

Centro de Ciencia del Clima y la Resiliencia. 2015. La megasequía 20102015: Una lección para el futuro. Santiago de Chile: Informe a la nación, 2015. 
Echeñique, Jorge. «El caso de Chile». 2012. En Dinámicas del Mercado de la Tierra en América Latina y el Caribe, editado por Fernando Soto y Sergio Gómez, 145-178. Santiago de Chile: FAO, 2012

Elden, Stuart. 2010. «Land, terrain, territory». Progress in Human Geography 34 (6): 799-817.

Escobar, Arturo. 2014. Sentipensar con la Tierra. Nuevas lecturas sobre desarrollo, territorio y diferencia. Cauca, Colombia: Ediciones UNAULA, 2014.

Haesbaert, Rogério. 2011. El Mito de la Desterritorialización: Del "fin de los territorios" a la multiterritorialidad. Ciudad de México: Editorial Siglo XXI, 2011.

Haesbaert, Rogério. 2014. Viver no Limite. Território e multitransterritorialidade em tempos de in-segurança e contenção. Rio de Janeiro: Bertrand Brasil, 2014.

Larraín, Sara. 2006. «El agua en Chile: entre los derechos humanos y las reglas del mercado». Revista Polis 14: 1-20. Acceso el 10 de mayo de 2006. http://polis.revues.org/5091

Larsimont. Robin y Virginia Grosso. 2014. «Aproximación a los nuevos conceptos híbridos para abordar las problemáticas hídricas». Cardinalis 2: $27-48$.

Lefebvre, Henri. 2013. La producción del espacio. Madrid: Editora Capital Swing, 1974.

Linton, Jamie y Jessica Budds. 2014. «The hydrosocial cycle: Defining and mobilizing a relational-dialectical approach to water». Geoforum 57: 170-180.

Lopes de Souza, Marcelo. 2013. Os conceitos fundamentais da pesquisa sócio-espacial. Rio de Janeiro: Bertrand Brasil, 2013.

Martínez-Alier, Joan. 2014. El ecologismo de los pobres. Conflictos ambientales y lenguajes de valoración. Santiago de Chile: Quimantú, 2005.

Marx, Karl. 1996. O Capital. Crítica da economia política. São Paulo, Editora Nova Cultural, 1867. 
Ministerio del interior. 2015. Política Nacional para los Recursos Hídricos 2015. Santiago de Chile: Delegación Presidencial para los Recursos Hídricos, Gobierno de Chile

Montañez, Gustavo y Ovidio Delgado. 1998. «Espacio, Territorio y Región: Conceptos básicos para un proyecto nacional». Cuadernos de Geografía 7 (1-2): 120-134.

Núñez, Jorge; Koen Verbist, Jim Wallis, Mel Schaefer, Luis Morales y Wim Cornelis. 2011. «Regional frequency analysis for mapping drought events in north-central Chile». Journal of Hydrology, 405 (3-4): 352366.

Peña, Humberto. 2016. «Desafíos de la seguridad hídrica en América Latina y el Caribe». CEPAL Serie Recursos Naturales e Infraestructura 178.

Porto-Gonçalves, Carlos Walter. 2002. «Da geografia às geo-grafias: um mundo em busca de novas territorialidades». En La Guerra Infinita: Hegemonía y terror mundial, editado por Emir Sader y Ana Ester Ceceña. Buenos Aires, CLACSO.

Porto-Gonçalves, Carlos Walter. 2003. Relatório técnico final. Projeto de pesquisa Geografia dos Conflitos Sociais na América Latina e Caribe. Brasilia: Conselho Nacional de Desenvolvimento Científico (CNPq), 2003.

Porto-Gonçalves, Carlos Walter. 2017. De Utopias e de Topoi: espaço e poder desde algumas experiências de lutas sociais na América Latina. La Paz: IPDRS, 2017.

Prieto, Manuel. 2016. «Transando el agua, produciendo territorios e identidades indígenas: el modelo de aguas chileno y los atacameños de Calama». Revista Estudios Sociales 55: 88-103. doi:10.7440/res55.2016.06.

Raffestin, Claude. 1993. Por uma geografia do poder. São Paulo: Editora Ática, 1980.

Ratzel, Friedrich. 1990. «Geografia do homem (Antropogeografia)». En Ratzel organizado por Antônio Carlos Moraes. São Paulo: Editora Ática, 1990. 
Romero Toledo, Hugo; Hugo Romero Aravena y Ximena Toledo. 2009 «Agua, poder y discursos en el conflicto socio-territorial por la construcción de represas hidroeléctricas en la Patagonia Chilena». Anuario de Estudios Americanos 66(2): 81-103.

Santos, Milton. 1994. «O retorno do território». En Território: globalização e fragmentação organizado por Milton Santos, Maria Adélia de Souza y Maria Laura Silveira. São Paulo: Hucitec, 1994.

Santos, Milton. 2006. A natureza do espaço. Técnica e Tempo. Razão e Emoção. São Paulo: Editora USP, 1994.

Seoane, José; Emilio Taddei y Clara Algranati. 2008. «El concepto "movimiento social" a la luz de los debates y la experiencia latinoamericana recientes». En Proyecto Conceptos y fenómenos fundamentales de nuestro tiempo coordinado por Pablo González Casanova. Ciudad de México: UNAM, 2008.

Skewes, Juan y Marta Silva. 2007. «Elementos para una comprensión ecológico-cultural de las narraciones míticas acerca de las aguas y los seres del agua en la cosmovisión mapuche huilliche». En Sociedad y Cultura: Reflexiones Transdisciplinarias editado por Claudia Smith, Neil. 2002. «Geografía, diferencia y las políticas de escala». Revista Terra Livre 19: 127-146.

Swyngedouw, Eric. 2009. «The Political Economy and Political Ecology of the Hydro-Social Cycle». Journal of Contemporary Water Research \& Education 142: 56-60.

Torres, Robinson; Gerardo Azócar, Noelia Carrasco y Mauricio Zambrano. Tatiana Costa y Bob Bolin. 2016. «Desarrollo forestal, escasez hídrica, y la protesta social mapuche por la justicia ambiental en Chile». Revista Ambiente \& Sociedade, 9(1): 121-146.

Ulloa, Astrid. 2011. «Concepciones de la naturaleza en la antropología actual». En Cultura y Naturaleza editado por Leonardo Montenegro. Bogotá: Jardín Botánico de Bogotá, 2011.

Vargas, Ramón y Nidia Piñeyro. 2005. El Hidroscopio. Ciudad de México: PNUMA, 2005. 
Yacoub, Cristina; Bibiana Duarte y Rutgerd Boelens. 2015. Agua y Ecología Política: El extractivismo en la agroexportación, la minería y las hidroeléctricas en Latinoamérica. Ecuador: Red Justicia Hídrica y Ediciones Abya-Yala. 